\title{
Effects of the Porous Microstructure on the Drag Coefficient in Flow of a Fluid with Pressure-Dependent Viscosity
}

\author{
M.S. Abu Zaytoon \\ Department of Mathematics, University of Petra, Amman, Jordan \\ S. Jayyousi Dajani \\ Department of Mathematics and Computer Science \\ Lake Forest College, Lake Forest, IL 60045, USA \\ M.H. Hamdan* \\ Department of Mathematics and Statistics \\ University of New Brunswick, Saint John, N.B., Canada, E2L 4L5 \\ hamdan@unb.ca *Corresponding Author
}

Received: February 17, 2021. Revised: August 13, 2021. Accepted: August 30, 2021. Published: September 1, 2021.

\begin{abstract}
Equations governing the flow of a fluid with pressure-dependent viscosity through an isotropic porous structure are derived using the method of intrinsic volume averaging. Viscosity of the fluid is assumed to be a variable function of pressure, and the effects of the porous microstructure are modelled and included in the pressuredependent drag coefficient. Five friction factors relating to five different microstructures are used in this work.
\end{abstract}

\section{Keywords - Pressure-dependent viscosity, drag coefficient, porous microstructure.}

\section{INTRODUCTION}

THE flow of fluids with pressure-dependent 1 viscosities through porous media has a host of applications in both old and emerging industries and processes. These include carbon sequestration in enhanced oil recovery groundwater flow and filtration problems, lubrication theory, and the pharmaceutical industry, [1-8]. These and many other important applications underscore the need to accurately model the flow phenomena, derive the appropriate governing equations, and provide solutions to initial and boundary value problems.

While the concept of fluid viscosity dependence on pressure can be traced back to the nineteenth century and the works of Stokes [9] and Barus [10, 11], modelling the flow of pressure-dependent viscosity fluids through porous media in quite recent and can be traced back to the work of Rajagopal [12] and coworkers (cf. [13-19], and the references therein).

The modelling aspect of this type of flow through porous media represents a paradigm shift with emphasis on the role high pressures and high pressure gradients play in porous media and their effects of interacting forces between flowing fluids and the solid matrix structures. The classical models of flow through porous media have been generalized in order to better handle high pressure phenomena, as discussed in what follows.

It has been argued that the celebrated Darcy's equation in the study of some flows through porous media suffers limitations that include its inability to account for microscopic inertia that arises due to tortuosity of the flow path, [20]. The absence of a viscous shear term in Darcy's equation imposes limits on its ability to account for viscous shear effects that arise when a macroscopic, solid boundary is encountered. Darcy's equation may be valid in low permeability, low porosity media where variations at the microscopic, pore-level length scale are negligible at the macroscopic length scale.

Nakshatrala and Rajagopal [2] discussed other limitations of Darcy's equation and its short-comings in predicting important phenomena in flow through porous media. They [2] emphasized that while Darcy's equation merely predicts fluid flux through the porous structure, 
its "flux prediction is not accurate at high pressures and pressure gradients".

In order to overcome some of the above limitations, Savarotova and Rajagopal [15], Nakshatrala and Rajagopal [2] provided a generalized form of Darcy's equation in which the drag coefficient depended on pressure. Chang et. al. [1] reported both Darcy's equation and Forchheimer's equation in the following generalized forms:

Generalized Darcy's Equation:

$\alpha(p, \vec{x}) \vec{u}+\nabla p=\rho \vec{g}$

with

$\alpha(p, \vec{x})=\frac{\mu_{0}}{k(\vec{x})} \cdot \exp \left[\beta_{B} p\right]$

Generalized Forchheimer's Equation:

$\alpha(\vec{u}, p, \vec{x}) \vec{u}+\nabla p=\rho \vec{G}$

with

$\alpha(\vec{u}, p, \vec{x})=\frac{\mu(p)}{k(\vec{x})}+\beta_{f}\|\vec{u}\|=\frac{\mu_{0}}{k(\vec{x})} \cdot \exp \left[\beta_{B} p\right]+\beta_{f} \rho\|\vec{u}\|$

wherein $\mu_{0}$ is the fluid fixed viscosity, $\beta_{B}$ is the experimentally obtained Barus coefficient, and $\beta_{F}$ is the Forchheimer or inertial coefficient. It is customary to write $\beta_{F}=\frac{C_{F}}{\sqrt{k(x)}}$, where $C_{F}$ is the Ergun coefficient with value between 0.375 and 0.5 .

In 1947, Brinkman [21] derived an extension to Darcy's equation by including a viscous term that would account for the viscous shear effects, which are important in a thin boundary layer near a macroscopic, solid boundary. Brinkman's equation, which describes the steady flow of a Newtonian fluid through a porous medium composed of a swarm of particles fixed in space, is written here in the following form

$-\nabla p+\mu_{e} \nabla^{2} \vec{u}-\frac{\mu}{\eta} \vec{u}+\rho \vec{g}=\overrightarrow{0}$

where $\vec{u}$ is the ensemble-averaged velocity, $\mu$ is the base fluid viscosity, $\mu_{e}$ is the viscosity of the fluid saturating the porous medium (i.e. effective viscosity), $\eta$ is the permeability and $p$ is the pressure.
The term $\frac{\mu}{\eta} \vec{u}$ is the Darcy resistance, and $\mu_{e} \nabla^{2} \vec{u}$ is the viscous shear term. It is clear that when $\mu_{e}=0$, Brinkman's equation (2) reduces to the following Darcy's equation, namely:

$-\nabla p-\frac{\mu}{\eta} \vec{u}+\rho \vec{g}=\overrightarrow{0}$

Generalization of Brinkman's equation for flow of fluids with pressure-dependent viscosity in porous media has been provided by Rajagopal [12], Srinivasan and Rajagopal [17], using homogenization, mixture theory, and thermodynamic balance. Complete and thorough analysis of all aspects of the generalized Brinkman's equation, together with its various forms, can be found in $[12,17]$.

In this work, we write the generalized Brinkman's equation in the following form, [18]:

$-\nabla p+\nabla \cdot \vec{T}-\alpha(p) \vec{u}+\rho \vec{g}=\overrightarrow{0}$

wherein

$\vec{T}=\mu\left(\nabla \vec{u}+(\nabla \vec{u})^{T}\right)$

where $\alpha(p)$ denotes the pressure-dependent drag coefficient and $\rho$ is the density of the fluid. Subramanian and Rajagopal [19] rightfully state that "the viscosity $\mu(p)$ is a measure of the frictional resistance in between fluid layers while $\alpha(p)$ is a measure of the friction between the fluid and the solid, at the pore".

Equation (7) does not explicitly take into account the porous medium microstructure or permeability of the medium, nor does it distinguish between one porous medium and another.

One way to distinguish between different porous structures is to experiment with various forms of $\alpha(p)$ and select the most representative form. Another possible way is the objective of the current work, where we take into account the porous microstructure in the drag coefficient. One could then experiment with $\alpha(p)$ to eliminate forms that are not suitable for a given microstructure.

In order to accomplish this, we start with the NavierStokes equations for flow of a fluid with pressuredependent viscosity, and use intrinsic volume averaging to obtain an averaged form of the equations, valid for flow through porous media. The surface integral that 
gives rise to the drag coefficient is analyzed and approximated using expressions for the porous microstructure. The effects of the porous microstructure will be accounted for using mathematical idealizations of the pore structure, based on the concept of Representative Unit Cell (RUC) that was introduced by Du Plessis and Masliyah, [22, 23] and the geometric factors of $\mathrm{Du}$ Plessis , [24] and of Du Plessis and Diedericks, [25]. Five different porous microstructures are discussed

\section{MODEL EQUATIONS}

The Averaging Approach

Consider the steady Navier-Stokes flow of an incompressible fluid with pressure-dependent viscosity. Flow is governed by the equations of continuity and momentum, expressed as, [5]:

$$
\begin{aligned}
& \nabla \cdot \vec{u}=0 \\
& \rho \nabla \cdot \vec{u} \vec{u}=-\nabla p+\nabla \cdot \vec{T}+\rho \vec{g} \\
& \vec{T}=\mu\left(\nabla \vec{u}+(\nabla \vec{u})^{T}\right)
\end{aligned}
$$

$\vec{u}$ is the velocity vector field, $p$ is the pressure, $\rho$ is the fluid density, $\mu=\mu(p)$ is the pressure-dependent viscosity of the fluid.

When the flow domain is a porous medium we seek a macroscopic form of the above equations, obtained by averaging the governing equations over a control volume, $V$, referred to as a Representative Elementary Volume (REV), [22, 23]. An REV is composed of a fluid-phase contained in the pore space, $V_{\varphi}$, and a solidphase contained in the porous matrix solid of volume $V_{s}$. The no-slip velocity condition, $\vec{u}=\overrightarrow{0}$, holds on the solid matrix.

Porous microstructure interactions with the flowing fluid are accounted for through an idealization of the pore geometry and the concept of a Representative Unit Cell (RUC), introduced in [22, 23], defined as the minimal REV that embeds average properties of the porous medium.

The fraction of pore space in the REV is the same as pore space fraction in the whole porous medium, thus having the same porosity as the medium. Porosity, $\varphi$, of the REV, hence that of the porous medium, is defined as, [26]:
$\varphi=\frac{1}{V} \int_{V} \mathcal{K}_{\varphi} d V=\frac{1}{V} \int_{V_{\varphi}} 1 d V=\frac{V_{\varphi}}{V}$

where $\mathcal{K}_{\varphi}$ is a fluid-phase function defined at position $\vec{x}$ in $V$ by:

$\mathcal{K}_{\varphi}(\vec{x})=\left\{\begin{array}{cc}1 ; & \vec{x} \in V_{\varphi} \\ 0 ; & \vec{x} \in V_{s}\end{array}\right.$

In what follows, we assume that porosity is a variable function of position. If porosity is constant, we simply factor it out from the resulting equations.

In order to average governing equations, we first define the volumetric phase average of a fluid quantity $F$ per unit volume, as:

$<F>=\frac{1}{V} \int_{V} \mathcal{K}_{\varphi} F d V=\frac{1}{V} \int_{V_{\varphi}} F d V$

and the intrinsic phase average (or the volumetric average of $F$ over the effective pore space, $V_{\varphi}$ ) as:

$<F>_{\varphi}=\frac{1}{V_{\varphi}} \int_{V} \mathcal{K}_{\varphi} F d V=\frac{1}{V_{\varphi}} \int_{V_{\varphi}} F d V$

Relationship between the volumetric phase average and the intrinsic phase average can be seen from equations (14), and (15), and the definition of porosity, (13), as:

$<F>=\varphi<F\rangle_{\varphi}$

and the deviation of an averaged quantity from its true (microscopic) value is given by the quantity

$F^{\circ}=F-<F>_{\varphi}$

Using the above definitions of averages, we can write the averaging rules in the following forms, listed here for ease of reference.

Letting $F$ and $H$ be volumetrically additive scalar quantities, $\vec{F}$ a vector quantity, and $c$ a constant (whose average is itself), then, [26]:

$$
<c F>=c<F>=c \varphi<F>_{\varphi}
$$

$$
\left\langle\nabla F>=\varphi \nabla<F>_{\varphi}+\frac{1}{V} \int_{S} F^{*} \vec{n} d S\right.
$$

where $S$ is the surface area of the solid matrix in the $\mathrm{REV}$ that is in contact with the fluid, and $\vec{n}$ is the unit normal vector pointing into the solid.

$$
<F \mp H>=\varphi<F>_{\varphi}+\varphi<H>_{\varphi}
$$




$$
\begin{aligned}
& <F H>=\varphi\langle F\rangle_{\varphi}\langle H\rangle_{\varphi}+\varphi\left\langle F^{\circ} H^{2}\right\rangle_{\varphi} \\
& \langle\nabla \cdot \vec{F}\rangle=\nabla \cdot \varphi\langle\vec{F}\rangle_{\varphi}+\frac{1}{V} \int_{S} \vec{F} \cdot \vec{n} d S
\end{aligned}
$$

(vi)... Due to the no-slip condition, a surface integral is zero if it contains the fluid velocity vector explicitly.

These averaging theorems are applied to equations (9) and (10) to obtain, respectively:

$$
\begin{aligned}
& \nabla \cdot \varphi<\vec{u}>_{\varphi}+\frac{1}{V} \int_{S} \vec{u} \cdot \vec{n} d S=0 \\
& \rho \nabla \cdot \varphi<\vec{u}>_{\varphi}<\vec{u}>_{\varphi}+\rho \nabla \cdot \varphi<\vec{u} \vec{u}>_{\varphi}+ \\
& \frac{\rho}{V} \int_{S} \vec{u} \vec{u} \cdot \vec{n} d S=-\varphi \nabla<p>_{\varphi}-\frac{1}{V} \int_{S} p^{\circ} \vec{n} d S \\
& +\rho \varphi<\vec{g}>_{\varphi}+\nabla \cdot \varphi<\vec{T}>_{\varphi}+\frac{1}{V} \int_{S} \vec{T} \cdot \vec{n} d S
\end{aligned}
$$

Equations (18) and (19) represent the intrinsic volume averaged continuity and momentum equations. The deviation terms and surface integrals appearing in the averaged equations contain information on the interactions between the fluid-phase and solid-phase in the REV. These are analyzed in what follows.

\section{Analysis of the Deviation Terms and Surface Integrals}

Using Gauss' divergence theorem, together with continuity equation (9), and averaging rule (vi), the surface integrals $\frac{1}{V} \int_{S} \vec{u} \cdot \vec{n} d S$ in (18) and $\frac{\rho}{V} \int_{S} \vec{u} \vec{u} \cdot \vec{n} d S$ in (19) vanish. Equation (9) thus takes the following final averaged form:

$\nabla \cdot \varphi<\vec{u}\rangle_{\varphi}=0$

The volume filter, or inertial deviation term, $\nabla \cdot \varphi\left\langle\vec{u}^{s} \vec{u}^{s}\right\rangle_{\varphi}$, is related to the hydrodynamic dispersion of the average velocity. Hydrodynamic dispersion through porous media is the sum of mechanical dispersion due to tortuosity of the flow path within the porous microstructure, and molecular diffusion arising from diffusion of fluid vorticity, [22,26]. The above deviation term is an inertial term representative of mechanical dispersion. Using rule (iv), we can write:

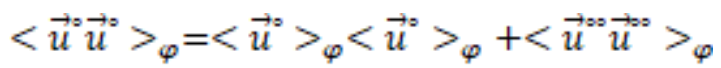

In the absence of high velocity and high porosity gradients, the deviations in the velocity vector are small, and the product of deviations (leading term on righthand-side of (21)) is small. Hence, the term $\boldsymbol{\nabla} \cdot \varphi\left\langle\vec{u} \vec{u} \vec{u}^{*}\right\rangle_{\varphi}$ is negligible, [26].

The term $\nabla \cdot \varphi<\vec{T}>_{\varphi}$ of equation (11) can be written as $\nabla \cdot \varphi<\mu \vec{I}\rangle_{\varphi}$, where $\vec{I}=\left(\nabla \vec{u}+\nabla \vec{u}^{T}\right)$, and expanded into the form

$$
\begin{aligned}
& \nabla \cdot \varphi\langle\mu \vec{I}\rangle_{\varphi}=\nabla \cdot \varphi\langle\mu\rangle_{\varphi}\langle\vec{I}\rangle_{\varphi}+ \\
& \nabla \cdot \varphi\left(<\mu^{3}>_{\varphi}<\vec{I}^{3}>_{\varphi}+<\mu^{\infty s} \vec{I}^{3+}>_{\varphi}\right)
\end{aligned}
$$

The last term on the right-hand-side of (22) involves products of deviations of the average fluid velocity gradients, $\left\langle\overrightarrow{I^{b}}\right\rangle_{\varphi}$, and deviations of the average viscosity, $\left\langle\mu^{\circ}\right\rangle_{\varphi}$. Assuming that these deviations are small in the absence of high velocity and viscosity gradients, the products of these small deviations can be ignored. We can then write:

$$
\nabla \cdot \varphi<\vec{T}>_{\varphi}=\nabla \cdot \varphi<\mu>_{\varphi}<\vec{I}>_{\varphi}
$$

The term $\frac{1}{V} \int_{S} p^{\otimes} \overrightarrow{\boldsymbol{n}} d S$ represents pressure fluctuations on the fluid-solid interface. In case of flow of a fluid with constant viscosity, Le Bars and Grae Worster [27] argue that this term is small, hence can be neglected. However, in case of high pressure flow through porous media, it may be of significance and could influence fluid-solid friction at the pore.

As recognized and discussed by Le Bars and Grae Worster [27] for the case of constant viscosity, the term

$\frac{1}{V} \int_{S} \vec{T} \cdot \vec{n} d S$ is the interfacial viscous stress exchange, which corresponds to the microscopic momentum exchange of the Newtonian fluid with the solid matrix. This term depends on

a) the morphology of the porous matrix,

b) the viscosity of the fluid (hence depends on pressure, for fluids with pressure-dependent viscosities) and,

c) if present, on the relative velocity of the fluidphase and the solid-phase. Assuming the porous matrix is stationary and rigid, velocity of the solid is zero in the current study.

For the case of pressure-dependent viscosity, it is quite possible that $\frac{1}{V} \int_{S} p^{0} \overrightarrow{\boldsymbol{n}} d S$ is dominant, compared with $\frac{1}{V} \int_{S} \vec{T} \cdot \vec{n} d S$, in which case fluid-solid friction at the pore could be modelled as a function of pressure and 
the Darcy drag is independent of viscosity. If, on the other hand, $\frac{1}{V} \int_{S} \vec{T} \cdot \vec{n} d S$ is of significance (due to importance of momentum exchange of the fluid with the solid matrix), then the Darcy drag could be expressed in terms of both viscosity and pressure.

Clearly, the first of these two cases would be more in line with the idea that viscosity $\mu(p)$ is a measure of the frictional resistance in between fluid layers while $\alpha(p)$ is a measure of the friction between the fluid and the solid, at the pore. In either case, the surface integrals, $\frac{1}{V} \int_{S} p^{\Delta} \overrightarrow{\boldsymbol{n}} d S$ and $\frac{1}{V} \int_{S} \vec{T} \cdot \overrightarrow{\boldsymbol{n}} d S$ can be combined to form a surface filter $\left.\frac{1}{V} \int_{S} \overrightarrow{(T} \cdot \vec{n}-p^{\circ} \vec{n}\right) d S$, in the sense introduced by Whitaker, [28, 29], that involves the normal derivative of $\vec{u}$.

Since the solid porous matrix affects the fluid through the portion of the surface area of the solid that is in contact with it, this surface filter contains the information necessary to quantify the forces exerted on the flowing fluid by the porous matrix. This form of surface filter has been abundantly analyzed in the literature for the case of constant viscosity, and has been identified with the force that gives rise to Darcy resistance. Since viscosity is a function of pressure, we can write this integral in terms of a function of pressure in order to emphasize significance of pressure fluctuations at pore level:

$$
\frac{1}{V} \int_{S}-\left(p^{\circ} \overrightarrow{\boldsymbol{n}}-\overrightarrow{\boldsymbol{T}} \cdot \overrightarrow{\boldsymbol{n}}\right) d S=-f \lambda(p) \varphi<\overrightarrow{\boldsymbol{u}}>_{\varphi}
$$

wherein $f$ is a friction factor and $\lambda(p)$ is a function of pressure that is not necessarily related to the functional form of viscosity as a function of pressure.

The above surface integral, or the friction factor, $f$, is a shear force integral which accounts for the viscous drag effects (Darcy resistance) that predominate in the Darcy regime, that is, for small Reynolds number flow. It is the velocity-independent viscous shear geometric factor that depends on the geometry of the porous medium and gives rise to the Darcy resistance.

Assuming that viscous drag effect is the only frictional force present, and defining hydrodynamic permeability as, [25]:

$\eta=\frac{\varphi}{f}$ then as a first approximation, the following expression can be used for surface integral (24):

$\frac{1}{V} \int_{S}-\left(p^{\circ} \overrightarrow{\boldsymbol{n}}-\overrightarrow{\boldsymbol{T}} \cdot \overrightarrow{\boldsymbol{n}}\right) d S=$
$\left.-\frac{\varphi}{\eta} \lambda(p) \varphi<\vec{u}\right\rangle_{\varphi}=-\alpha(p, \vec{x}) \varphi\langle\vec{u}\rangle_{\varphi}$

where the Darcy drag term in (26) is given by:

$$
\alpha(p, \vec{x})=f \lambda(p)=\frac{\varphi}{\eta(\vec{x})} \lambda(p)
$$

Using (26), equation (19) can be written in the following form:

$$
\begin{aligned}
& \rho \nabla \cdot \varphi<\vec{u}>_{\varphi}<\vec{u}>_{\varphi}=-\varphi \nabla<p>_{\varphi} \\
& +\nabla \cdot \varphi\left\langle\vec{T}>_{\varphi}-\alpha(p, \vec{x}) \varphi<\vec{u}>_{\varphi}+\rho \varphi<\vec{g}>_{\varphi}\right.
\end{aligned}
$$

Equation (28) is the intrinsically averaged momentum equation (10). Porosity is left in this equation as a variable function of position. The momentum equation corresponding to constant porosity is easily recovered from (28) by factoring out $\varphi$, as follows. Denoting $\langle\vec{u}\rangle_{\varphi}, \quad\langle\vec{T}\rangle_{\varphi}, \quad\langle\vec{p}\rangle_{\varphi}$ and $\langle\vec{g}\rangle_{\varphi}$ by $\vec{u}$, $\vec{T}=\mu\left(\nabla \vec{u}+\nabla \vec{u} \vec{u}^{T}\right), \vec{p}$ and $\vec{g}$, respectively, equation (28) reduces to:

$\rho \nabla \cdot \vec{u} \vec{u}=-\nabla p+\nabla \cdot \vec{T}-\alpha(p, \vec{x}) \vec{u}+\rho \vec{g}$

while continuity equation is obtained from (20) as:

$\nabla \cdot \vec{u}=0$

For variable porosity, we let $\vec{q}=\varphi\langle\vec{u}\rangle_{\varphi}$ be the specific discharge, $\vec{G}=\varphi\langle\vec{g}\rangle_{\varphi}, \mu^{*}=\varphi\langle\mu\rangle_{\varphi}$ we write (20) in the following final form:

$\nabla \cdot \vec{q}=0$

and equation (28) in the following final form:

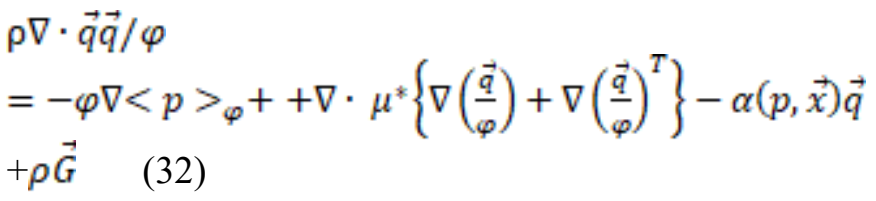

where $\alpha(p, \vec{x})$ is as given by (27).

\section{CHOICES FOR THE DRAG COEFFICIENT}

Equation (27) shows that we need $\lambda(p), \mu^{*}(p)$ and friction factor $f$ in order to determine a drag coefficient that takes into account the effects of the porous 
microstructure. Once a choice is made for $\alpha(p, \vec{x})$, model equations (31) and (32) are updated. In what follows, we list choices for $\lambda(p)$ and $\mu^{*}(p)$, and expressions for the friction factor and its values for different porosities.

\section{Choices for $\lambda(p)$ and $\mu^{*}(p)$}

Many choices exist for $\lambda(p)$ and $\mu^{*}(p)$ and have been used by various authors ( $c f$. Kannan and Rajagopal [18], Fusi et.al. [4] and the references therein). Typical among these are the Barus' relations for viscosity, [5], namely

$\mu(p)=\mu_{0} e^{\delta\left(p-p_{0}\right)}$

which is approximated for small $\delta$, or small pressure differences, by

$\mu(p)=\mu_{0}\left[1+\delta\left(p-p_{0}\right)\right]$

and the following for $\lambda(p),[18]$

$$
\lambda(p)=\lambda_{0} ; \lambda(p)=\lambda_{0}(1+\gamma p) ; \lambda(p)=\lambda_{0} e^{\gamma p}
$$

where $\lambda_{0}>0 ; \quad y>0$

\section{Porous Microstructure and Friction Factor}

Expressions for $f$ require a mathematical description of the porous matrix and its microstructure. Du Plessis and Diedericks, [25], carried out extensive analysis on evaluating these geometric factors for isotropic porous media, based on Du Plessis and Masliyah's concept of a Representative Unit Cell (RUC), [22, 23], which they defined as the minimal REV in which the average properties of the porous medium are embedded. For granular and consolidated isotropic porous media, the following expressions, [25], summarized and computed in Tables 1-7, are adopted in this work for $f$ and for the hydrodynamic permeability, $\eta$. We note that as $\varphi \rightarrow 1$, the porous medium approaches free-space and $f \rightarrow 0$, for all microstructures considered.

\begin{tabular}{|c|c|}
\hline Description & Geometric Factor \\
& $f$ \\
\hline Granular Matter & $36(1-\varphi)^{\frac{2}{3}}$ \\
\hline & $\frac{d^{2}\left[1-(1-\varphi)^{\frac{1}{2}}\right]\left[1-(1-\varphi)^{\frac{2}{2}}\right]}{}$ \\
\hline
\end{tabular}

\begin{tabular}{|l|l|}
\hline $\begin{array}{l}\text { Consolidated } \\
\text { Matter }\end{array}$ & $\frac{42.69(1-\tau)}{(\tau d)^{2} \varphi}$ \\
\hline $\begin{array}{l}\text { Unidirectional } \\
\text { Fibre Bed }\end{array}$ & $\frac{24 \sqrt{1-\varphi}}{d^{2}\left(1-\sqrt{1-\varphi)^{2}}\right.}$ \\
\hline $\begin{array}{l}\text { Ergun's } \\
\text { Equation }\end{array}$ & $\frac{150(1-\varphi)^{2}}{\varphi^{2}\left(d_{p}\right)^{2}}$ \\
\hline $\begin{array}{l}\text { Kozeny-Carman } \\
\text { Relation }\end{array}$ & $\frac{180(1-\varphi)^{2}}{\varphi^{2}\left(d_{m}\right)^{2}}$ \\
\hline
\end{tabular}

Table 1 Geometric Factor Expressions

\begin{tabular}{|c|c|}
\hline Description & $\begin{array}{c}\text { Hydrodynamic Permeability } \\
\qquad \eta=\frac{\varphi}{f}\end{array}$ \\
\hline $\begin{array}{l}\text { Granular } \\
\text { Matter }\end{array}$ & $\frac{d^{2} \varphi\left[1-(1-\varphi)^{\frac{1}{2}}\right]\left[1-(1-\varphi)^{\frac{2}{2}}\right]}{36(1-\varphi)^{\frac{2}{3}}}$ \\
\hline $\begin{array}{l}\text { Consolidated } \\
\text { Matter }\end{array}$ & $\frac{(\varphi \tau d)^{2}}{42.69(1-\tau)}$ \\
\hline $\begin{array}{l}\text { Unidirectional } \\
\text { Fibre Bed }\end{array}$ & $\frac{\varphi d^{2}(1-\sqrt{1-\varphi})^{2}}{24 \sqrt{1-\varphi}}$ \\
\hline $\begin{array}{l}\text { Ergun's } \\
\text { Equation }\end{array}$ & $\frac{\varphi^{3}\left(d_{p}\right)^{2}}{150(1-\varphi)^{2}}$ \\
\hline $\begin{array}{l}\text { Kozeny- } \\
\text { Carman } \\
\text { Relation }\end{array}$ & $\frac{\varphi^{3}\left(d_{m}\right)^{2}}{180(1-\varphi)^{2}}$ \\
\hline
\end{tabular}

Table 2 Hydrodynamic Permeability Expressions

\begin{tabular}{|l|l|}
\hline$\varphi$ & $\begin{array}{c}\text { Kozeny-Carman } \\
f=\frac{180(1-\varphi)^{2}}{\varphi^{2}\left(d_{m}\right)^{2}}\end{array}$ \\
\hline 0.5 & $180 / d_{m}^{2}$ \\
\hline 0.9 & $2.222222 / d_{m}^{2}$ \\
\hline
\end{tabular}




\begin{tabular}{|l|c|}
\hline 0.95 & $0.498615 / / d_{m}^{2}$ \\
\hline 0.99 & $0.02222222 / d_{m}^{2}$ \\
\hline 0.995 & $0.00454534 / d_{m}^{2}$ \\
\hline 0.999 & $0.00018036 / d_{m}^{2}$ \\
\hline 1 & 0 \\
\hline
\end{tabular}

Table 3 Geometric Factor Values Vs. Porosity

\begin{tabular}{|l|l|}
\hline$\varphi$ & $\begin{array}{c}\text { Ergun's Equation } \\
f=\frac{150(1-\varphi)^{2}}{\varphi^{2}\left(d_{p}\right)^{2}}\end{array}$ \\
\hline 0.5 & $150 / d_{p}^{2}$ \\
\hline 0.9 & $1.85185167 / d_{p}^{2}$ \\
\hline 0.95 & $0.4155125 / d_{p}^{2}$ \\
\hline 0.99 & $0.01851851 / d_{p}^{2}$ \\
\hline 0.995 & $0.00378778 / d_{p}^{2}$ \\
\hline 0.999 & $0.00015030 / d_{p}^{2}$ \\
\hline 1 & 0 \\
\hline
\end{tabular}

Table 4 Geometric Factor Values Vs. Porosity

\begin{tabular}{|l|l|}
\hline$\varphi$ & $\begin{array}{c}\text { Unidirectional Fibre } \\
\text { Bed }\end{array}$ \\
$f=\frac{24 \sqrt{1-\varphi}}{d^{2}(1-\sqrt{1-\varphi})^{2}}$ \\
\hline 0.5 & $197.824447 / d^{2}$ \\
\hline 0.9 & $16.232637 / d^{2}$ \\
\hline 0.95 & $8.902941 / d^{2}$ \\
\hline 0.99 & $2.9629630 / d^{2}$ \\
\hline 0.995 & $1.96514386 / d^{2}$ \\
\hline 0.999 & $0.80932342 / d^{2}$ \\
\hline 1 & 0 \\
\hline
\end{tabular}

Table 5 Geometric Factor Values Vs. Porosity

\begin{tabular}{|l|l|}
\hline$\varphi$ & Granular Matter \\
& $f=\frac{36(1-\varphi)^{\frac{2}{2}}}{d^{2}\left[1-(1-\varphi)^{\frac{1}{2}}\right]\left[1-(1-\varphi)^{\frac{2}{2}}\right]}$ \\
\hline 0.5 & $297.0774570 / d^{2}$ \\
\hline 0.9 & $18.44906 / d^{2}$ \\
\hline
\end{tabular}

\begin{tabular}{|l|l|}
\hline 0.95 & $8.95067 / d^{2}$ \\
\hline 0.99 & $2.233504000 / d^{2}$ \\
\hline 0.995 & $1.308021611 / d^{2}$ \\
\hline 0.999 & $0.404040404 / d^{2}$ \\
\hline 1 & 0 \\
\hline
\end{tabular}

Table 6 Geometric Factor Values Vs. Porosity

\begin{tabular}{|c|c|c|}
\hline \multirow[t]{2}{*}{$\varphi$} & \multicolumn{2}{|c|}{$\begin{array}{c}\text { Consolidated Matter } \\
\qquad \begin{array}{c}f=\frac{42.69(1-\tau)}{(\tau d)^{2} \varphi} \\
\tau \approx \frac{1+2 \varphi}{3}\end{array}\end{array}$} \\
\hline & $\tau$ & $f$ \\
\hline 0.5 & 0.5 & $170.76 / d^{2}$ \\
\hline 0.9 & 0.719 & $25.782926 / d^{2}$ \\
\hline 0.95 & 0.967 & $1.585855 / d^{2}$ \\
\hline 0.99 & 0.993 & $0.306119 / d^{2}$ \\
\hline 0.995 & 0.9967 & $0.1439733 / d^{2}$ \\
\hline 0.999 & 0.9993 & $0.0285265 / d^{2}$ \\
\hline 1 & $\overline{1}$ & 0 \\
\hline
\end{tabular}

Table 7 Geometric Factor Values Vs. Porosity

In the above Tables, tortuosity is approximated by:

$$
\tau \approx \frac{1+2 \varphi}{3}
$$

and $d$ is a microscopic characteristic length, $d_{p}$ is the average pore diameter in a channel-like porous material, and $d_{m}$ is a median diameter of spherical particles

\section{CONCLUSION}

In this work, intrinsic volume averaging was implemented in deriving equations governing the flow of an incompressible fluid with variable, pressuredependent viscosity through isotropic porous media. Five porous microstructures were incorporated in the drag coefficient in order to distinguish between porous structures. This work might prove to be of value in the determination of which pressure function to use for a given porous configuration.

For flow through a constant porosity medium, governing equations are (29) and (30), with drag coefficient given by (27). For flow through variable porosity media, governing equations are (31) and (32), with drag coefficient given by (27). In both momentum equations (29) and (32), we left in convective terms. 


\section{REFERENCES}

[1] J. Chang, K.B. Nakashatrala and J.N. Reddy, "Modification to Darcy-Forchheimer model due to pressure-dependent viscosity: consequences and numerical solutions", J. Porous Media, vol. 20(3), pp. 263-285, 2017.

[2] K.B. Nakshatrala and K.R. Rajagopal, "A numerical study of fluids with pressure-dependent viscosity flowing through a rigid porous medium", Int. J. Numer. Meth. Fluids, vol. 67, pp. 342-368, 2011.

[3] P.W. Bridgman, The Physics of High Pressure. MacMillan, New York, 1931.

[4] L. Fusi, A. Farina and F. Rosso, "Mathematical models for fluids with pressure- dependent viscosity flowing in porous media", International Journal of Engineering Science, vol. 87, pp. 110-118, 2015.

[5] K.D. Housiadas, G.C. Georgiou and R.I. Tanner, "A note on the unbounded creeping flow past a sphere for Newtonian fluids with pressure-dependent viscosity”, Int. Journal of Engineering Science, vol. 86, pp. 1-9, 2015.

[6] F.J. Martinez-Boza, M.J. Martin-Alfonso, C. Callegos and M. Fernandez, "High-pressure behavior of intermediate fuel oils", Energy Fuels, vol. 25, pp. 5138-5144, 2011.

[7] A.Z. Szeri, Fluid Film Lubrication: Theory and Design, Cambridge University Press, 1998.

[8] P.H. Vergne, "Pressure viscosity behavior of various fluids", High Press. Res., vol. 8, pp. 451-454, 1991.

[9] G.G. Stokes, "On the theories of the internal friction of fluids in motion, and of equilibrium and motion of elastic solids", Trans. Camb. Philos. Soc., vol. 8, pp. 287-305, 1845.

[10] C.J. Barus, "Note on dependence of viscosity on pressure and temperature". Proceedings of the American Academy, vol. 27, pp. 13-19, 1891.

[11] C.J. Barus, "Isothermals, isopiestics and isometrics relative to viscosity", American Journal of Science, vol. 45, pp. 87-96, 1893.

[12] K.R. Rajagopal, "On a hierarchy of approximate models for flows of incompressible fluids through porous solids", Mathematical Models and Methods in the Applied Sciences, vol. 17 (2), pp. 215-252, 2007.

[13] J. Hron, J., Malek, and K.R. Rajagopal, "Simple flows of fluids with pressure-dependent viscosities", Proceedings of the Royal Society, vol. 457, pp. 16031622, 2001.

[14] K.R. Rajagopal, G. Saccomandi and L. Vergori, "Flow of fluids with pressure- and shear-dependent viscosity down an inclined plane", Journal of Fluid Mechanics, vol. 706, pp. 173-189, 2012.

[15] V.L. Savatorova and K.R. Rajagopal, "Homogenization of a generalization of Brinkman's equation for the flow of a fluid with pressure dependent viscosity through a rigid porous solid", ZAMM, 91(8), pp. 630-648, 2011.

[16] S. Srinivasan, A. Bonito and K.R. Rajagopal, "Flow of a fluid through a porous solid due to high pressure gradient", Journal of Porous Media, vol. 16, pp. 193203, 2013.
[17] S. Srinivasan and K.R. Rajagopal, "A thermodynamic basis for the derivation of the Darcy, Forchheimer and Brinkman models for flows through porous media and their generalizations", International Journal of NonLinear Mechanics, vol. 58, pp. 162-166, 2014.

[18] K. Kannan and K.R. Rajagopal, "Flow through porous media due to high pressure gradients", J. Applied Mathematics and Computation, vol. 199, pp. 748-759, 2008.

[19] S.C. Subramanian and K.R. Rajagopal, "A note on the flow through porous solids at high Pressures". Computers and Mathematics with Applications, vol. 53, pp. 260-275, 2007.

[20] N. Rudraiah, "Coupled Parallel Flows in a Channel and a Bounding Porous Medium of Finite Thickness", J. Fluids Engineering, ASME, vol. 107, pp. 322-329, 1985.

[21] H.C. Brinkman, "A Calculation of the viscous force exerted by a flowing fluid on a dense swarm of particles", Appl. Scientific Res., vol. A1, pp. 27-34, 1947.

[22] J. P. Du Plessis and J. H. Masliyah, "Mathematical modeling of flow through consolidated isotropic porous media," Transport in Porous Media, vol. 3, pp. 145-161, 1988.

[23] J. P. Du Plessis and J. H. Masliyah, "Flow through isotropic granular porous media," Transport in Porous Media, vol. 6, pp. 207-221, 1991.

[24] J. P. Du Plessis, 1994, "Analytical quantification of coefficients in the Ergun equation for fluid friction in a packed bed," Transport in Porous Media, vol. 16, pp. 189-207, 1994.

[25] J. P. Du Plessis and G. P. J. Diedericks, "Pore-Scale Modeling of Interstitial Phenomena," In: Fluid Transport in Porous Media, J.P. Du Plessis, ed., Computational Mechanics Publications, Southampton, 1997, pp. 61104.

[26] M.S. Abu Zaytoon, F.M. Allan, T.L. Alderson and M.H. Hamdan, "Averaged equations of flow of fluid with pressure-dependent viscosity through porous media”, Elixir Appl. Math., vol. 96, pp. 4133641340, 2016.

[27] M. Le Bars and M. Grae Worster, "Interfacial conditions between a pure fluid and a porous medium: implications for binary alloy solidification", Journal of Fluid Mechanics, vol. 550, pp.149-173, 2006.

[28] S. Whitaker, "Volume Averaging of Transport Equations," In: Fluid Transport in Porous Media, J.P. Du Plessis, ed., Computational Mechanics Publications, Southampton, 1997, pp. 1-60.

[29] S. Whitaker, "The method of volume averaging," Kluwer Academic Publishers, Dordrecht, 1999. 


\section{Contribution of individual authors:}

All authors reviewed the literature and conducted averaging process.

M.S. Abu Zaytoon and S. Jayyousi Dajani provided the tables and calculations, and contributed to the writing of some parts of the mathematical analysis.

M.H. Hamdan provided analysis and wrote most of the manuscript.

\section{Sources of funding:}

There are no sources of funding for this work.

\section{Creative Commons Attribution License 4.0 (Attribution 4.0 International, CC BY 4.0)}

This article is published under the terms of the Creative Commons Attribution License 4.0

https://creativecommons.org/licenses/by/4.0/deed.en_US 